\title{
Particle-based Simulation of Blood Flow and Vessel Wall Interactions in Virtual Surgery
}

\author{
Jing Qin \\ Department of Diagnostic \\ Radiology, \\ National University of \\ Singapore, Singapore \\ dnraj@nus.edu.sg
}

\author{
Wai-Man Pang \\ Spatial Media Group, \\ Computer Arts Lab, \\ University of Aizu, Japan \\ wmpang@u-aizu.ac.jp
}

\author{
Binh P. Nguyen \\ School of Information and \\ Communication Technology, \\ Hanoi University of \\ Technology, Vietnam \\ binhnp@it-hut.edu.vn
}

\author{
Dong $\mathrm{Ni}$ \\ Department of Biomedical \\ Engineering, \\ Shenzhen University, \\ Shenzhen, P.R. China \\ dni@cse.cuhk.edu.hk
}

\author{
Chee-Kong Chui \\ Department of Mechanical \\ Engineering, \\ National University of \\ Singapore, Singapore \\ mpecck@nus.edu.sg
}

\begin{abstract}
We propose a particle-based solution to simulate the interactions between blood flow and vessel wall for virtual surgery. By coupling two particle-based techniques, the smoothed particle hydrodynamics (SPH) and mass-spring model (MSM), we can simulate the blood flow and deformation of vessel seamlessly. At the vessel wall, particles are considered as both boundary particles for SPH solver and mass points for the MSM solver. We implement an improved repulsive boundary condition to simulate the interactions. The computation of blood flow dynamics and vessel wall deformations are performed in an alternating fashion in every time step. To ensure realism, parameters of both SPH and MSM are carefully configured. Experimental results demonstrate the potential of the proposed method in providing real-time and realistic interactions for virtual vascular surgery systems.
\end{abstract}

\section{Categories and Subject Descriptors}

I.6 [Simulation and Modeling]: Applications

\section{General Terms}

Algorithms

\section{Keywords}

Smoothed Particle Hydrodynamics, Blood Flow and Vessel Wall Interactions, Virtual Surgery

\section{INTRODUCTION}

Permission to make digital or hard copies of all or part of this work for personal or classroom use is granted without fee provided that copies are not made or distributed for profit or commercial advantage and that copies bear this notice and the full citation on the first page. To copy otherwise, to republish, to post on servers or to redistribute to lists, requires prior specific permission and/or a fee.

SoICT'10, August 27-28, 2010, Hanoi, Vietnam.

Copyright 2010 ACM 978-1-4503-0105-3/10/08 ...\$10.00.
Physically based modeling and simulation is central to virtual reality based surgical training and planning systems. It holds out the promise of realistically simulating mechanical and physiological behaviors of human tissues, and complicated interactions between these tissues and virtual surgical instruments. However, while considerable efforts have been dedicated to develop deformation models for soft tissues and haptic models for real-time interactions between these tissues and virtual instruments, little attention is given to the simulation of fluid and deformable tissue interactions, such as those between blood flow and vessel wall.

In reality, interactions of blood flow and vessel wall profoundly influence the quality of many surgical training and planning systems. For example, in endovascular aneurysm repair(EVAR), aneurysm rupture is in principle believed to occur when the mechanical stress acting on the wall exceeds the strength of the wall tissue. Therefore, knowledge of the stress distribution in an intact aneurysm wall could be a biophysical basis for assessing its risk of rupture and making a better surgery planning $[19,5]$. In other surgical procedures such as hysteroscopic simulators, the diffusion of intra-uterine bleeding and floating tissue fragments should obscure the view of the surgeons in real surgery. In these cases, realistically simulation of blood flow and its interactions with surrounding tissues can help surgeons learn how to perform correct actions during interventions [24].

However, it is a challenging task to simulate the interactions between blood flow and vessel wall in a real-time manner in virtual surgery systems. In computer graphics and virtual reality, previous works usually use Eulerian methods for fluids and Lagrangian methods for solids, e.g. taking the solid velocity as a boundary condition for the fluid solver while integrating the fluid pressure with the solid boundary to exert a force on the solid $[21,6]$. However, it usually encounters problems such as low performance, instability, and uncontrollable leakage. These shortcomings limit the application of these methods in virtual surgery systems.

In this paper, we propose a comprehensive particle-based method for simulation of blood flow and vessel wall inter- 
actions in virtual surgery. We use smoothed particle hydrodynamics (SPH) to simulate the blood flow while using mass-spring model (MSM) to simulate the deformation of vessel wall. At the boundary, the particles are considered as both boundary particles for SPH solver and mass points for the MSM solver. We implement an improved repulsive boundary condition to simulate the interactions between blood flow and vessel wall, e.g., when flow particles approach boundary particles, analogous to inter-molecular forces, the boundary particles exert central forces on fluid particles. If the integrated force exerted on a spring exceeds its maximum stretch force, the spring breaks and the vessel wall is ruptured. We use an interleaved fashion to solve the dynamics of blood flow and vessel wall separately in every time step. To ensure realism, parameters of both SPH and MSM are carefully configured. Experiment results demonstrate that the proposed method has potential to provide real time and realistic interactions for virtual vascular surgery systems.

The rest of the paper is organized as follows. We review related work in Section 2, work out our particle based method in Section 3, give the implementation details of the improved repulsive boundary condition in Section 4, provide experiment results in Section 5 and draw conclusions in Section 6.

\section{RELATED WORK}

In the past, the adoption of physically based models is limited by the difficulties in modeling blood flow and the performance of the computational power. The development of computational techniques in fluid dynamics [18], together with the increasing capability of the hardware, finds promising applications in computer graphics and virtual reality applications. Many physically-based approaches have been developed to simulate the behavior of fluids $[2,3,25]$ as well as the interactions between fluids and rigid or deformable solids $[1,21]$. But the main disadvantage of most of these mesh-based methods is computational complexity. This prohibits their applications in interactive surgical simulations.

Among these physically based methods, particle-based solutions are popular in real-time fluid simulation due to their independence of complicated mesh structures and relatively higher performance. The first work that adopted particle systems in computer graphics was to model a class of fuzzy objects[20]. Afterward, particle systems have been widely used to simulate waterfalls, ship wakes and breaking waves. Smoothed Particle Hydrodynamics (SPH) is known as one of efficient particle based methods, which was introduced simultaneously by Gingold and Monaghan [7], and Lucy [11], for the simulation of astrophysical problems such as fission and stars. Refer to [10] for a comprehensive introduction as well as recent developments of SPH. This technique was first introduced to computer graphics community to depict fire and other gaseous phenomena [22]. This method is adopted by Müller et. al. to simulate blood for virtual surgery [14]. Later, our research group based on a hardware accelerated $\mathrm{SPH}$ for bleeding simulation in many different surgical training system [16, 17]. However, many of these works did not provide solutions for interactions between blood and deformable tissues. Even Müller et. al. have reported their further attempts on simulating interactions of fluids with deformable solids [15]. Unfortunately, how the solids are deformed by the pressure of fluids are not clearly described. In this work, we aim at integrating SPH with MSM to simulate the interactions between blood flow and vessel wall in a real time manner.

\section{COUPLING BLOOD FLOW AND VESSEL WALL}

\subsection{SPH Solver for Momentum Equation of Blood Flow}

In general, the basic equations of blood flow motion are derived by imposing that the fluid conserves mass, momentum and energy. We assume the temperature of the blood flow is nearly constant and there is no heat input into the system. Therefore, the equation for the balance of energy is the same as the balance of mechanical energy and work, and the energy conservation leads to no new independent equation. In addition, the particle system can inherently conserve mass by keeping both the number of particles and the mass of each particles constant. Thus, we can focus on how to solve the Navier-Stokes which formulates the conservation of momentum:

$$
\rho \frac{\partial \vec{V}}{\partial t}=-\rho \vec{V} \cdot \nabla \vec{V}+\mu \nabla^{2} \vec{V}-\nabla p+\rho \vec{g}
$$

Here $\rho$ is the density; $\vec{V}$ is the vector velocity field; the vector operator $\nabla$ is defined as $\nabla \equiv \vec{i} \frac{\partial}{\partial x}+\vec{j} \frac{\partial}{\partial y}+\vec{k} \frac{\partial}{\partial z} ; p$ is the pressure; $\vec{g}$ means the body forces such as gravitational forces; $\mu$ is the viscosity coefficient. Based on Eq. 1, we need to calculate force density caused by $\operatorname{viscosity}\left(\mu \nabla^{2} \vec{V}-\rho \vec{V}\right.$. $\nabla \vec{V})$, pressure $(-\nabla p)$ and external force $(\rho \vec{g})$ in every time step.

Compared to other fluid simulation approaches, $\mathrm{SPH}$ is relatively simple in implementation and fast in computation. The basic mechanism of SPH is based on the following equation:

$$
A_{S}(\vec{x})=\sum_{j} m_{j} \frac{A_{j}}{\rho_{j}} W(\vec{r}, h)
$$

where a scalar quantity $A$ at position $\vec{x}$ is a weighted sum of contributions from neighboring particles. $m_{j}$ and $\rho_{j}$ are the mass and density of particle $j$, respectively, and $\vec{r}=\vec{x}-\overrightarrow{x_{j}}$. $A_{j}$ is the field quantity at $\vec{x}_{j}$. The function $W(\vec{r}, h)$ is referred to the smoothing kernel with the properties $\int W(\vec{r}, h) d \vec{r}=$ 1 and $\lim _{h \rightarrow 0} W(\vec{r}, h)=\delta(\vec{x})$, where $h$ is the support radius and $\delta$ is the Dirac-function.

Initially, particles have three quantities including mass, position and velocity. In every time step of the simulation, SPH is used to evaluate the density $\left(\rho_{i}\right)$ and the force density $\left(f_{i}^{p}, f_{i}^{v}, f_{i}^{e x}\right)$ caused by pressure, viscosity and external forces at particle $i$. We calculate the density and pressure force density based on the equations derived in[14]:

$$
\begin{gathered}
\rho_{i}=\sum_{j} m_{j} W(\vec{r}, h), \\
f_{i}^{p}=-\nabla p\left(r_{i}\right)=-\sum_{j} m_{j}\left(\frac{p_{j}+p_{i}}{2 \rho_{j}}\right) \nabla W(\vec{r}, h)
\end{gathered}
$$

In our current implementation, we consider gravitational force as the only external force, which can be calculated by:

$$
f_{i}^{e x}=\rho_{i} g
$$




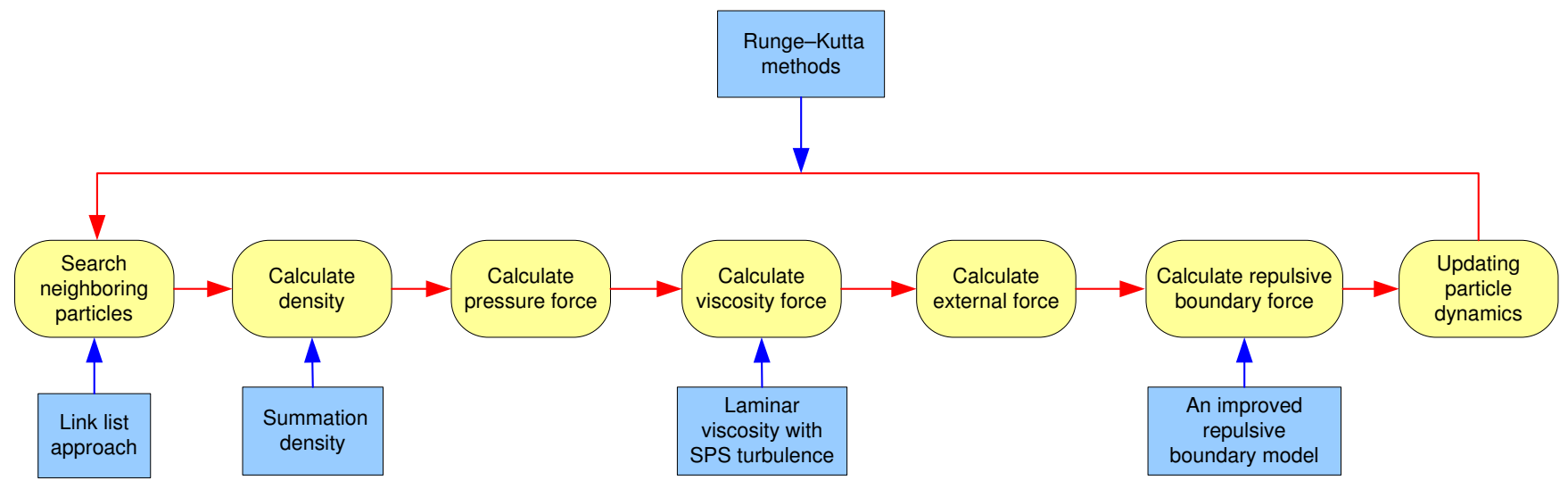

Figure 1: The flow chart of our SPH implementation.

As blood cannot be simply considered as laminar flow, especially when it flows through a small caliber orifice or a irregular vessel shape such as aneurysm, we integrated laminar viscosity and sub-particle scale(SPS) approach [8] to calculate the viscosity force density:

$f_{i}^{v}=\sum_{j} \rho_{j} \frac{4 \mu \vec{r}\left(\vec{V}_{j}-\vec{V}_{i}\right)}{\left(\rho_{i}+\rho_{j}\right)|\vec{r}|^{2}} \nabla W(\vec{r}, h)+\sum_{j} \rho_{j}\left(\frac{\tau_{j}}{\rho_{j}^{2}}+\frac{\tau_{i}}{\rho_{i}^{2}}\right) \nabla W(\vec{r}, h)$

Please refer to [4] for the details of the equation derivation.

\subsection{Implementation of SPH Solver}

Fig. 1 shows the flow chart of our implementation of SPH. In every time step, we first search the neighboring particles for every particle based on the given radius. Typically, there are three kinds of methods to search the neighboring particles: all pair approach, link list approach and tree based approach. In our implementation, we employ the link list approach proposed in [12], which is much faster than all pair approach and has a higher degree of parallelism than tree based approach.

Also, there are two methods for calculating the density: summation density method, which directly applies the SPH approximations to the density itself, and continues density method, which approximates the density according to the continuity equation using the concepts of SPH approximations plus some transformations [9]. Although summation density method may cause some boundary effects leading to inaccuracy results at the boundary, it is more popular in practical applications of SPH, as it can exactly conserve total mass but continues density method can not. In our implementation, we alleviate the boundary effects by setting some virtual fluid particles around the boundary when calculating the density.

After calculating the pressure force density, the viscosity force density, and external density based on the equations in Section 3.1, we employ an improved repulsive boundary condition to simulate the interactions between blood flow and vessel wall, which will be introduced in detail in the following section. Finally, we employ Runge-Kutta method as time integration scheme to calculate dynamic properties of both blood flow and vessel wall.

\section{REPULSIVE BOUNDARY CONDITION}

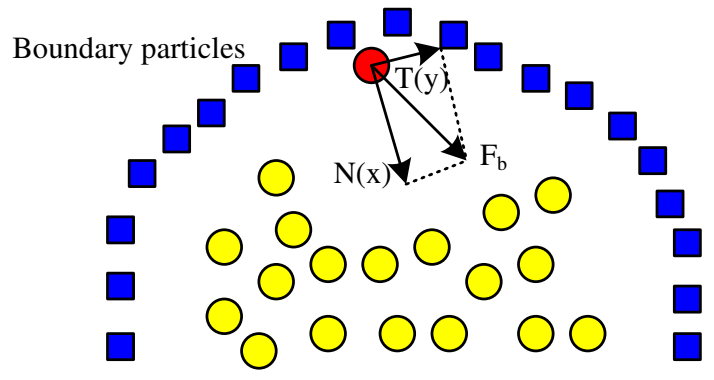

Fluid particles

Figure 2: Improved repulsive boundary condition.

We implement an improved repulsive boundary condition to simulate the interactions between blood flow and vessel wall. The basic idea is to produce a force normal to the boundary from neighboring boundary particles to the approaching fluid particles without lowering its velocity in tangent direction [13]. Thus, the force on a fluid particle from a boundary particle has two components: alone the normal and tangent directions respectively, as shown in Fig. 2. Obviously, we need to adopt different schemes to deal with the two force components.

The repulsive boundary force can be written as following equation:

$$
F_{b}=\vec{n} N(x) T(y)
$$

where $N(x)$ and $T(y)$ are forces in normal and tangent directions, $\vec{n}$ is a unit vector representing the direction of the force, and $x$ and $y$ are the distances between the boundary particle and the fluid particle in normal and tangent directions, respectively. The form of $N(x)$ should fulfill three requirements: (1) when the distance between the fluid particle and the boundary particle exceeds a threshold $D_{f v}, N(x)$ should equal to $0,(2)$ when the fluid particle approaches the boundary particle, $N(x)$ is inversely proportional to the distance between them and (3)if $N(x)$ exceeds the maximum stretch force $f_{\text {smax }}$ of the spring connected to the boundary particle, the spring breaks and $N(x)$ falls to 0 at the mo- 


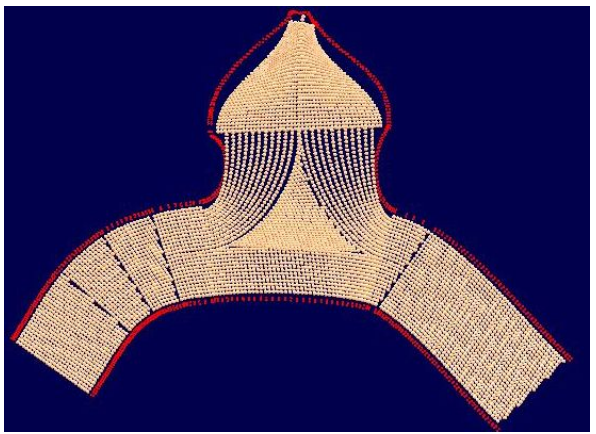

(a)

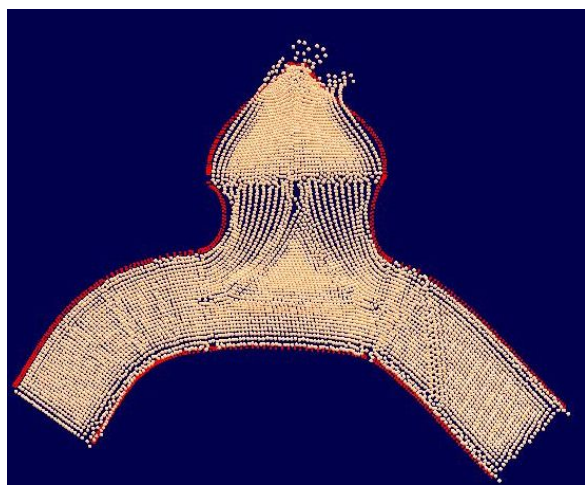

(c)

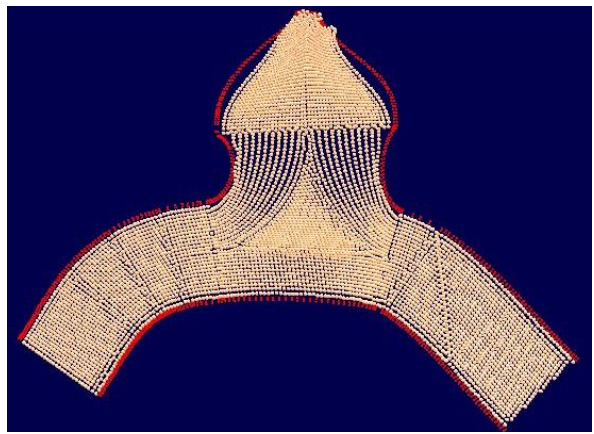

(b)

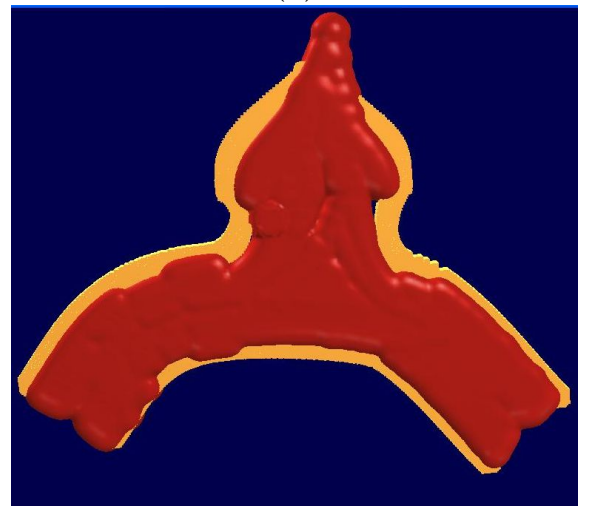

(d)

Figure 3: An aneurysm model with 6961 particles: (a) the initial status, (b) after 20 time steps, particles flow to the cavity of the aneurysm, (c) after 50 time steps, particles gush out from vessel wall, indicating the rupture of the aneurysm, and (d) the rupture of the aneurysm rendered based on Marching Cubes method.

ment. In this paper, we employ the following equation to compute $N(x)$ :

$$
N(x)= \begin{cases}0 & \text { if } x>D_{f v} \\ \Psi \frac{1}{\sqrt{r}}(1-r) & \text { if } 0<x<D_{f v} \& N(x)<f_{\text {smax }} \\ 0 & \text { if } 0<x \| N(x)>f_{\text {smax }}\end{cases}
$$

where $r=\frac{x}{2 h}$ and $h$ is support radius of SPH. The parameter $\Psi$ is set based on the equation described in [13].

On the other hand, the function $\mathrm{T}(\mathrm{y})$ is designed to ensure that when a fluid particle moves parallel to the curve of boundary, the total forces generated from these boundary particles along the tangent direction should maintain constant. Thus, the fluid particle can smoothly flow alone the boundary.

\section{RESULTS}

In order to evaluate the feasibility of the proposed method, we carried out a series of experiments. First, we assess the time performance of the method with various numbers of particles. Second, we construct an aneurysm model to demonstrate the improved repulsive boundary condition. Third, we conduct a set of experiments to investigate the effects of key parameters on the performance of our method. All experiments were conducted on a desktop $\mathrm{PC}$ equipped with Intel $i 5-750$ (2.66GHz, quad-core), $4 G$ RAM and NVIDIA Quadro FX 3800 display adapter.

One of the advantages of particle-based method is it has relatively higher performance compared with mesh-based solutions. Table 1 shows the results of the time performance experiments. It is observed that the frame rate decreases when the number of particles increases. When there are around 5000 fluid particles and 600 boundary particles, the frame rate can keep at around $20 \mathrm{fps}$, which can fulfill the requirement of most of interactive virtual surgery systems. When the particle number increases to around 10000, the frame rate is less than $10 \mathrm{fps}$. In these experiments, we set time step length $\delta t$ as $0.1 s$, support radius $h$ as 0.01 , the search radius $r$ as 0.02 and the distance threshold $D_{f v}$ as 0.02 .

Table 1: The time performance of various numbers of particles

\begin{tabular}{|c|c|c|}
\hline Fluid particles & Boundary particles & Frame rate (fps) \\
\hline 500 & 80 & 52.1 \\
\hline 1000 & 80 & 51.8 \\
\hline 1500 & 80 & 50.8 \\
\hline 2000 & 200 & 45.1 \\
\hline 2500 & 200 & 39.3 \\
\hline 3000 & 300 & 35.5 \\
\hline 3500 & 300 & 31.2 \\
\hline 4000 & 600 & 23.7 \\
\hline 5000 & 600 & 18.2 \\
\hline 8000 & 1000 & 11.1 \\
\hline 10000 & 1000 & 5.7 \\
\hline
\end{tabular}

Fig. 3 shows an example of our method: an aneurysm model with 6961 particles. Fig. 3(a) shows the initial status 


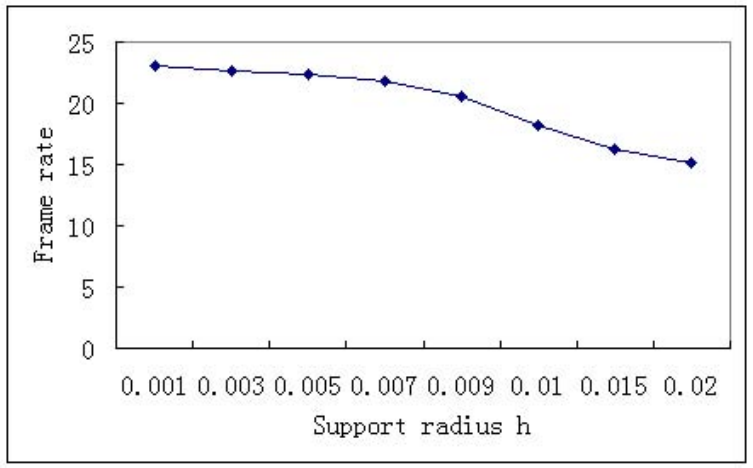

Figure 4: Plotting of support radius h vs. time performance.

of the aneurysm. Fig. 3(b) shows the status after 20 time steps, when more and more particles flow into the cavity of the aneurysm. After 50 time steps, when the repulsive forces between the boundary particles and the fluid particles exceeds the maximum stretch force of some springs at the boundary, the particles gush out from the vessel wall, indicating the rupture of the aneurysm. In this experiment, we set time step length $\delta t$ as $0.1 s$ and support radius $h$ as 0.01 .

It is noticed that some key parameters may greatly influence the performance of the method. As we always set the search radius $r$ and the distance threshold $D_{f v}$ based on the support radius $h, h$ is one of the most important parameters in our method. Fig. 4 shows the changes of frame rate with various values of $h$, where 5000 fluid particles and 600 boundary particles are involved and time step length $\delta t$ is $0.1 s$. It is observed the performance degrades when $h$ increases. This is because the number of neighboring particles increases with the increasing of $h$, leading to more computations in every time step. However, the accuracy of SPH will be partly lost if $h$ is too small to provide enough neighboring particles. In practice, we need to find a balance value based on specific requirement. Similar to $h$, a trade-off need to be made when setting $\delta t$ to keep the balance between speed and accuracy.

\section{DISCUSSION AND CONCLUSION}

We proposed a particle based solution to simulate the interactions between blood flow and vessel wall for virtual surgery. Experiment results demonstrate that the proposed method has potential to provide real time and realistic interactions for virtual vascular surgery systems. There are some questions that need to be further addressed. First, it is observed that when Marching Cubes based rendering method is employed, the performance greatly deceases. We will aim at integrating a GPU-based Marching Cubes [23] to maintain the performance after rendering. We will also work on implementing the proposed method on GPU to achieve better performance as well as further assessing its robustness and effectiveness. More important, we will integrate our method into some virtual vascular surgery systems to further demonstrate its feasibility.

\section{Acknowledgment}

This research has been supported in part by a research grant from A*STAR Biomedical Research Council and Singapore Bioimaging Consortium and graduate school research grant of University of Aizu (No. G-9).

\section{REFERENCES}

[1] C. Batty, F. Bertails, and R. Bridson. A fast variational framework for accurate solid-fluid coupling. ACM Trans. Graph., 26(3):100, 2007.

[2] M. Carlson, P. J. Mucha, V. H. I. R.B., and G. Turk. Melting and flowing. In Proceedings of the 2002 ACM SIGGRAPH Symposium on Computer Animation, pages 167-174, July 2002.

[3] M. Carlson, P. J. Mucha, and G. Turk. Rigid fluid: animating the interplay between rigid bodies and fluid. ACM Transaction on Graphics, 23(3):377-384, Aug 2004.

[4] R. Dalrymple and B. Rogers. Numerical modeling of water waves with the sph method. Coastal Engineering, 53:141-147, 2006.

[5] M. F. Fillinger, M. L. Raghavan, S. P. Marra, J. L. Cronenwett, and F. E. Kennedy. In vivo analysis of mechanical wall stress and abdominal aortic aneurysm rupture risk. J. Vasc. Surg., 36(3):589-597, 2002.

[6] O. Génevaux, A. Habibi, and J.-M. Dischler. Simulating fluid-solid interaction. In Graphics Interface, pages 31-38, 2003.

[7] R. A. Gingold and J. J. Monaghan. Smoothed particle hydrodymamics theory and application to non-spherical stars. Royal Astronomical Society, Monthly Notices, 181(11):375-389, 1997.

[8] H. Gotoh, S. Shao, and T. Memita. Sph-les model for numerical investigation of wave interaction with partially immersed breakwater. Coastal Engineering Journal, 46(1):39-63, 2004.

[9] G. Liu and M. Liu. Smoothed particle hydrodynamics: a meshfree particle method. World Scientific Publishing Co. Pte. Ltd, 5 Toh Tuck Link, Singapore, 2003.

[10] M. B. Liu and G. R. Liu. Smoothed particle hydrodynamics (sph): an overview and recent developments. Archives of Computational Methods in Engineering, 17(1):25-76, 2010.

[11] L. B. Lucy. A numerical approach to the testing of the fission hypothesis. The Astronomical Journal, 82(12):1013-1024, 1977.

[12] J. Monaghan and J. Lattanzio. A refined method for astrophysical problems. Astron. Astrophys, 149:135-143, 1985.

[13] J. J. Monaghan and A. Kos. Solitary waves on a cretan beach. Journal of Waterway, Port, Coastal, and Ocean Engineering.

[14] M. Müller, S. Schirm, and M. Teschner. Interactive blood simulation for virtual surgery based on smoothed particle hydrodynamics. Journal of Technology and Health Care, 12(1):25-31, 2004.

[15] M. Müller, S. Schirm, M. Teschner, B. Heidelberger, and M. Gross. Interactions of fluids with deformable solids. Journal of Computer Animaiton and Virtual Worlds, 15(3-4):159-171, 2004.

[16] W.-M. Pang, J. Qin, Y.-P. Chui, T.-T. Wong, K.-S. 
Leung, and P.-A. Heng. Orthopedics surgery trainer with ppu-accelerated blood and tissue simulation. In Medical Image Computing and Computer-Assisted Intervention, pages 842-849, 2007.

[17] J. Qin, Y.-P. Chui, W.-M. Pang, K.-S. Choi, and P.-A. Heng. Learning blood management in orthopedic surgery through gameplay. IEEE Computer Graphics and Applications, 30:45-57, 2010.

[18] A. Quarteroni, M. Tuveri, and A. Veneziani. Computational vascular fluid dynamics: problems, models and methods. Computing and Visualization in Science, 2(4):163-197, 2000.

[19] M. L. Raghavan, D. A. Vorp, M. P. Federle, M. S. Makaroun, and M. W. Webster. Wall stress distribution on three-dimensionally reconstructed models of human abdominal aortic aneurysm. J. Vasc. Surg., 31(4):760-769, 2000.

[20] W. T. Reeves. Particle systems - a technique for modeling a class of fuzzy objects. ACM Transaction on Graphics, 2(2):91-108, Apr. 1983.

[21] A. Robinson-Mosher, T. Shinar, J. Gretarsson, J. Su, and R. Fedkiw. Two-way coupling of fluids to rigid and deformable solids and shells. ACM Trans. Graph. $27(3), 2008$
[22] J. Stam and E. Fiume. Depicting fire and other gaseous phenomena using diffusion processes. In Computer Graphics Proceedings, Annual Conference Series, 1995 (ACM SIGGRAPH '95 Proceedings), pages 129-136, 1995.

[23] Y. Xie, P. A. Heng, G. Wang, and T.-T. Wong. Gpu-friendly marching cubes for visualizing translucent isosurfaces. In Proceedings of Medicine Meets Virtual Reality, pages 500-503, 2007.

[24] J. Zátonyi, R. Paget, G. Székely, and M. Bajka. Real-time synthesis of bleeding for virtual hysteroscopy. In Proceedings of Medical Image Computing and Computer-Assisted Intervention MICCAI 2003, volume 1, pages 67-74, November 2003.

[25] Y. Zhu and R. Bridson. Animating sand as a fluid. ACM Transaction on Graphics(Proc. of ACM SIGGRAPH 2005), 24(3):965-972, July 2005. 